\title{
Assessment of forecast skill of high- and coarse-resolution numerical weather prediction models in predicting visibility/ fog over Delhi, India
}

\author{
Aditi $^{1, *}$ and Raghavendra Ashrit ${ }^{2}$ \\ ${ }^{1}$ Ministry of Earth Sciences, Lodi Road, New Delhi 110 003, India \\ ${ }^{2}$ National Centre for Medium Range Weather Forecasting, Noida 201 309, India
}

Accurate forecasts of visibility are important to avoid disruption in air and highway traffic caused due to the formation of dense fog. However, accurate forecasting of visibility/fog remains a challenge as the genesis and development of fog is a result of many processes. In view of this, models have been developed in recent years to forecast visibility and the occurrence of fog is measured in terms of visibility. The global Unified Model of the National Centre for Medium Range Weather Forecasting, known as NCUM, provides direct output of visibility. As aviation is severely affected at the Indira Gandhi International Airport, New Delhi, India, a high-resolution model was set up to forecast visibility over the airport. The present study analyses the performance of the coarse-resolution global model and high-resolution model in predicting visibility over Delhi. Visibility is categorized into three ranges - very poor $(0-200 \mathrm{~m})$, poor $(200-1000 \mathrm{~m})$ and clear conditions beyond $1 \mathrm{~km}$. The accuracy of forecast in different ranges of visibility is determined using different statistical scores. Evaluation of the results shows that the performance of both high and coarse resolution model remains low in poor visibility conditions. Though the high-resolution model performs better than the coarse-resolution model in predicting a drop in visibility, it also has higher number of false alarms. None of the model is able to predict the very poor visibility conditions. The prediction of visibility from the high-resolution model can further be improved by inclusion of real-time aerosol fields in the model.

Keywords: Aerosol, forecast skill, visibility, fog, numerical weather prediction model.

FOG formation in the northern plains of India, including parts of Haryana, Punjab and Bihar is a common phenomenon every year during December and January. Low visibility conditions due to fog cause diversions, delays and flight cancellations at airports in these different states result in substantial economic losses. Such scenario has been an attraction for both the weather and aviation fore-

*For correspondence. (e-mail: aditi.singh76@gov.in) caster in India over the years and numerous studies have been conducted to highlight the socio-economic concerns due to fog ${ }^{1}$. Most of the studies conducted in the past focused on the formation of $\operatorname{fog}^{2-5}$. Some specific studies have been attempted in recent years to predict fog over the northern plains of India using different approaches such as statistical ${ }^{6,7}$, numerical weather prediction (NWP) models $^{8}$ and diagnostic approach ${ }^{9,10}$.

As accurate prediction of fog from the NWP models remains challenging due to its small spatial and temporal scales, efforts have been made worldwide to obtain visibility as a direct output of the model ${ }^{11,12}$. The visibility forecasts from these models are not obtained explicitly, but derived from other meteorological parameters such as cloud water content, relative humidity and precipitation ${ }^{13}$. The operational unified model (UM) of the United Kingdom Meteorological Office (UKMO) was used to predict visibility and aerosol content ${ }^{14}$. The diagnosis of visibility within the model depends on the humidity and aerosol content.

The UM was adopted as the global operational model by the National Centre for Medium Range Weather Forecasting (NCMRWF), India, in 2012 and is known as NCUM. The pioneering attempt to predict fog using forecast of visibility from NCUM was carried out in 2014. Visibility forecasts from NCUM were utilized to predict fog over the northern plains of India ${ }^{15}$. The study concluded that although NCUM is able to capture the spatial extent of fog over any region, the forecasted values of visibility remain high in comparison to those based on the observations. Further efforts were made to analyse the reason for high values of visibility from NCUM ${ }^{16}$. The study concluded that due to fixed value of aerosol content, the model fails to correctly predict visibility over any location. A high-resolution Delhi Model (DM) was developed to predict visibility over the Indira Gandhi International Airport (IGIA), New Delhi ${ }^{17}$. The performance of DM was tested for some specific cases of fog over Delhi. However, an extensive study on the performance of high-resolution DM in predicting visibility and fog over Delhi for one complete season of fog is still 
RESEARCH ARTICLES

Table 1. Differences between NCUM-G, NCUM-R and DM

\begin{tabular}{|c|c|c|c|}
\hline Science options & NCUM-G & NCUM-R & DM \\
\hline Initial condition & Data assimilation $^{18}$ & $\begin{array}{l}17 \mathrm{~km} \text { initial condition downscaled } \\
\text { to } 1.5 \mathrm{~km}\end{array}$ & $\begin{array}{l}1.5 \mathrm{~km} \text { initial condition downscaled } \\
\text { to } 330 \mathrm{~m}\end{array}$ \\
\hline Horizontal grid length & $17 \mathrm{~km}$ & $1.5 \mathrm{~km}$ & $330 \mathrm{~m}$ \\
\hline Vertical levels & $70($ model top $80 \mathrm{~km})$ & 70 (model top $40 \mathrm{~km})$ & 70 (model top $40 \mathrm{~km})$ \\
\hline Model time-step & $7.5 \mathrm{~min}$ & $1 \mathrm{~min}$ & $12 \mathrm{sec}$ \\
\hline PBL scheme & Unstable 1D BL scheme ${ }^{26}$ & $\begin{array}{l}\text { 1D BL scheme }{ }^{26} \text { combined with 3D } \\
\text { Smagorinsky scheme (blended } \\
\text { PBL scheme) }\end{array}$ & $\begin{array}{l}\text { 1D BL scheme }{ }^{26} \text { combined with 3D } \\
\text { Smagorinsky scheme (blended } \\
\text { PBL scheme) }\end{array}$ \\
\hline Convection scheme & Mass flux convection scheme & Convection scheme 'off' & Convection scheme 'Off' \\
\hline RHcrit & $\begin{array}{l}0.92 \text { at the surface and decreasing } \\
\text { to } 0.8 \text { at } 1 \mathrm{~km} \text {, constant above }\end{array}$ & $\begin{array}{l}0.97 \text { at the surface and decreasing to } \\
0.9 \text { at } 3.5 \mathrm{~km} \text {, constant above }\end{array}$ & $\begin{array}{c}0.97 \text { at the surface and decreasing to } \\
0.9 \text { at } 3.5 \mathrm{~km} \text {, constant above }\end{array}$ \\
\hline
\end{tabular}

lacking. An effort has been made in the present study to assess the performance of the high-resolution model in forecasting visibility over Delhi during winter months. The study aims to highlight the advantage of highresolution model over coarse-resolution model in predicting visibility during fog and clear conditions. The models are also assessed in terms of predicting meteorological conditions favourable for the formation of radiative fog.

\section{Model description}

The global model utilized in the study is the operational model of NCMRWF and is known as NCUM-G. The model has a horizontal resolution of $17 \mathrm{~km}$. There are 70 vertical levels with model top at $80 \mathrm{~km}$. The model timestep is $7.5 \mathrm{~min}$. The 4D-Var data assimilation scheme is used for analysis four times (00, 06, 12 and 18 UTC) a day $^{18}$. The dynamical core of the model consists of nonhydrostatic, fully compressible, deep atmospheric equations of motion discretized on a regular latitude/longitude grid using semi-implicit, semi-Lagrangian formulation ${ }^{19}$. The physical processes in the models are parameterized using different schemes such as radiation parameterization $^{20}$, large-scale precipitation ${ }^{21}$, boundary layer parameterization $^{22,23}$, cloud $^{24}$ and land surface scheme ${ }^{25}$. Visibility forecasts are available at an interval of $3 \mathrm{~h}$ every day for a forecast length of ten days.

The horizontal resolution of DM is $330 \mathrm{~m}$ and it covers a domain of $100 \mathrm{~km} \times 100 \mathrm{~km}$. The model is nested inside the regional model known as NCUM-R with a grid size of $1.5 \mathrm{~km}$. The initial and boundary conditions for NCUM-R are provided by NCUM-G.

The regional model is used to provide initial and boundary conditions for DM at a downscaled resolution (horizontal resolution $1.5 \mathrm{~km}$ ). The lateral boundary conditions to DM are updated every $15 \mathrm{~min}$ and run for forecast length of $36 \mathrm{~h}$ based on 00 UTC initial conditions. Visibility forecasts are available at an interval of $1 \mathrm{~h}$ from DM.
Besides grid length, there are other differences between the models (Table 1). The turbulence parameterization scheme in NCUM-G is the $1 \mathrm{D}$ boundary layer (BL) scheme $^{26}$, whereas a blended PBL scheme ${ }^{27}$ in which the $1 \mathrm{D}$ boundary layer scheme is combined with 3D Smagronisky turbulence scheme ${ }^{28}$ is used in NCUM$\mathrm{R}$ and DM. This scheme is used in the high-resolution models because at short grid lengths, turbulence does not remain sub-grid and the model starts to resolve the large eddies. This implies that in a high-resolution model the grid scale becomes similar to dominant turbulence length scale and the dominant modes of BL turbulence are partially resolved by the model. These resolutions are referred as the grey zone of BL turbulence ${ }^{29}$ and the fundamental assumptions of turbulence are no longer valid $^{30,31}$. A local 3D sub-grid diffusion scheme is used to parameterize the turbulence at these scales. A new BL parameterization scheme was proposed ${ }^{27}$, which can be used across the entire range of resolution. The scheme blends between 3D Smagronisky turbulence scheme and 1D boundary layer scheme.

The critical relative humidity (RHcrit) is higher in the two high-resolution models (NCUM-R and DM) than in NCUM-G. RHcrit is the value of relative humidity at which cloud will start to form and is used for cloud parameterization $^{24}$. The higher value in DM is used to simulate the low sub-grid cloud cover variability in highresolution models than coarse-resolution models. This is not essentially $100 \%$ in low-resolution models due to subgrid variability, but approaches $100 \%$ in high-resolution models as there is less sub-grid variability.

\section{Diagnosis of visibility}

The diagnosis of visibility in the models is related to the local scattering characteristics of the atmosphere ${ }^{32}$. The aerosol particles in the model get hydrated and become fog droplets based on the Kohler curves and atmospheric humidity. The hydrated aerosol is then used to calculate 
the scattering coefficient, which is converted to visibility forecast using an exponential scattering law ${ }^{33}$ given as

$$
\mathrm{Vis}=\frac{-\ln \varepsilon}{\beta_{\mathrm{air}}+\beta(\mathrm{RH}, m)} \text {. }
$$

$\varepsilon$ is the liminal constant set to $0.05, \beta_{\text {air }}$ the extinction coefficient due to clean air and $\beta(\mathrm{RH}, m)$ is the extinction coefficient due to aerosol particles and is a function of relative humidity $(\mathrm{RH})$ and mass mixing ratio $(m)$ of aerosols.

Aerosols in the NCUM-G, NCUM-R and DM are represented in terms of single aerosol mass-mixing ratio $(m)$ which is a fixed constant value. It is assumed that ammonium sulphate is the predominant aerosol. The aerosol number concentration is computed in terms of the mass mixing ratio of ammonium sulphate using eq (2) below. These aerosols are activated based on Kohler curves and relative humidity. Thus, the change in the number of aerosols in the model may have potential influence on fog forecasts directly through visibility calculation.

$$
N=N_{0}\left(\frac{m}{m_{0}}\right)^{1 / 2},
$$

where $N_{0}=5.0 \times 10^{8} \mathrm{~m}^{-3}$ corresponds to that of standard aerosol particles considered as ammonium sulphate. The standard mass mixing ratio $\left(m_{0}\right)$ is defined as

$$
m_{0}=\frac{4}{3} \pi \frac{\rho}{\rho_{a}} N_{0} r_{0}^{3},
$$

where $\rho$ is the density and $r_{0}$ is the radius of ammonium sulphate taken as $1700 \mathrm{~kg} \mathrm{~m}^{-3}$ and $1.6 \times 10^{-7} \mathrm{~m}$ respectively. $\rho_{a}$ is the density of air. A value of $10 \mu \mathrm{g} / \mathrm{kg}$ is used in NCUM-G, whereas a high value of $200 \mu \mathrm{g} / \mathrm{kg}$ is used in DM based on the sensitivity experiments.

\section{Observational data}

The plains of North India experience fog from the first week of November to February. However, fog over Delhi generally occurs for a maximum number of days in December and January. An earlier study ${ }^{34}$ utilized data of IGIA, for the period 1960-1998 and found that drop in visibility below $800 \mathrm{~m}$ is observed around 1430 UTC, which begins to improve around 0300 UTC in December and January. Thus, the visibility observations over IGIA $\left(28.56^{\circ} \mathrm{N}, 77.12^{\circ} \mathrm{E}\right)$ available from Meteorological Airport Report (METAR) were analysed from 12 UTC of a day to 09 UTC of the next day to identify clear and fog conditions during December 2017-February 2018. Visibility is divided into three categories, viz. clear, poor and very poor depending on the observed values. Different types of fog are identified based on the values of visibility $^{35}$. Clear conditions occur when observed visibility is greater than or equal to $1 \mathrm{~km}$ and there is no fog. Poor visibility conditions occur when the observed visibility is less than $1 \mathrm{~km}$ but greater than $200 \mathrm{~m}$ and corresponds to fog. Very poor visibility conditions occur when the observed visibility is less than or equal to $200 \mathrm{~m}$ and corresponds to dense fog. The number of observations in three months (December, January and February) during clear, poor and very poor visibility conditions was 513, 124 and 37 respectively. The observations of air temperature, dew point temperature and wind speed are also available from METAR.

\section{Methodology}

\section{Verification of visibility forecasts under three categories}

The forecast of visibility from NCUM-G and DM was compared with observations for 513, 124 and $37 \mathrm{~h}$ corresponding to clear, poor and very poor visibility conditions respectively. The forecast of visibility from NCUM-G is available at an interval of $3 \mathrm{~h}$. Thus, the three-hourly forecast of visibility from both the models was verified with observations. The observations from 12 UTC of a day to 09 UTC of the next day were compared with $12-33 \mathrm{~h}$ forecasts in the three categories of visibility during the three months.

The scores were computed for the three thresholds of visibility corresponding to clear, poor and very poor conditions using the contingency table (Tables 2 and 3). The values given in Table 2 are the number of correctly predicted non-occurrences $(c n)$, the number of missed occurrences $(m)$, the number of wrongly predicted occurrences or false alarms $(f a)$ and the number of correctly predicted occurrences or hits $(h)$.

The percentage correct (PC) gives the fraction of forecasts that are correct. The score is not well suited for high values of threshold as most of the cases are correctly predicted non-occurrences. The probability of detection/ hit rate (POD) shows the fraction of observed 'yes' events that are correctly forecasted. It is good for rare events as it ignores false alarms and is sensitive to hits. The false alarm rate (FAR) gives the percentage of predicted 'yes' events that are not actually observed. It is a measure of false predictions and is sensitive to false alarms, but ignores misses. The frequency bias (FBI) quantifies the over- and under-prediction of visibility for a given threshold and is a useful score for the evaluation of the model. The scores described above are defined as follows

$$
\mathrm{PC}=\frac{(h+c n)}{\text { Total }} \times 100
$$


Table 2. Contingency table for different thresholds of visibility. Observed (O), forecast (F)

\begin{tabular}{lllll}
\hline & \multicolumn{2}{c}{ NCUM-G } & DM \\
\hline Thresh1: Vis $>1 \mathrm{~km}$ & $\mathrm{O}<$ Thresh1 & $\mathrm{O} \geq$ Thresh1 & $\mathrm{O}<$ Thresh1 & $\mathrm{O} \geq$ Thresh1 \\
$\mathrm{F}<$ Thresh1 & $c n=06$ & $m=01$ & $c n=34$ & $m=21$ \\
$\mathrm{~F} \geq$ Thresh1 & $f a=155$ & $h=512$ & $f a=127$ & $h=492$ \\
Thresh2:Vis $<1 \mathrm{~km}$ & $\mathrm{O}<$ Thresh2 & $\mathrm{O} \geq$ Thresh2 & $\mathrm{O}<$ Thresh2 & $\mathrm{O} \geq$ Thresh2 \\
$\mathrm{F}<$ Thresh2 & $c n=546$ & $m=121$ & $c n=514$ & $m=107$ \\
$\mathrm{~F} \geq$ Thresh2 & $f a=04$ & $h=03$ & $f a=36$ & $h=17$ \\
Thresh3:Vis $\leq 200 \mathrm{~m}$ & $\mathrm{O}<$ Thresh3 & $\mathrm{O} \geq$ Thresh3 & $\mathrm{O}<$ Thresh3 & $\mathrm{O} \geq$ Thresh3 \\
$\mathrm{F}<$ Thresh3 & $c n=637$ & $m=37$ & $c n=635$ & $m=37$ \\
$\mathrm{~F} \geq$ Thresh3 & $f a=00$ & $h=00$ & $f a=02$ & $h=00$ \\
\hline
\end{tabular}

$C n$, Correct negatives; $m$, Misses; $h$, Hits; $f n$, False alarms.

Table 3. Mean error for observed and predicted values of visibility (Vis), relative humidity $(\mathrm{RH})$ and temperature $(\mathrm{T})$ at $1.5 \mathrm{~m}$ and wind speed $(\mathrm{W})$ at $10 \mathrm{~m}$

\begin{tabular}{lcc}
\hline & NCUM-G & DM \\
\hline Vis $(\mathrm{km})$ & 1.11 & 0.39 \\
$\mathrm{Rh}(\%)$ & -5.0 & -12.0 \\
$T\left({ }^{\circ} \mathrm{C}\right)$ & 0.76 & 0.75 \\
$W(\mathrm{~m} / \mathrm{s})$ & -0.23 & -1.00 \\
\hline
\end{tabular}

$$
\begin{aligned}
& \mathrm{POD}=\frac{h}{(h+m)}, \\
& \mathrm{FAR}=\frac{f a}{(f a+h)}, \\
& \mathrm{FBI}=\frac{(f a+h)}{(m+h)} .
\end{aligned}
$$

These scores are utilized to verify the forecasts and draw conclusions about forecast quality from the models. A perfect forecast should produce only the hits and correct negative events. These scores are also used to evaluate the types of error associated with the models.

\section{Verification of visibility forecasts during fog case studies}

Three days were selected from December 2017 and January 2018 when visibility started reducing below $1 \mathrm{~km}$ from midnight and increased to $1 \mathrm{~km}$ and beyond it in mid-morning or afternoon (Figure $1 a$ ). The two cases of 12-13 December 2017 and 23-24 January 2018 correspond to dense fog with poor visibility conditions. The case of 1-2 January corresponds to very dense fog with very poor visibility conditions from 18 to 00 UTC. Relative humidity was observed more than $90 \%$ during dense fog and close to $100 \%$ in very dense fog cases (Figure $1 \mathrm{~b}$ ). The decrease in temperature was observed for fog hours and lowest temperature was observed for the hours corresponding to very dense fog (Figure $1 c$ ). During dense and very dense fog wind speed was observed to be less than $2 \mathrm{~m} \mathrm{~s}^{-1}$. Thus, based on the typical onset (1800 UTC) and dissipation (06 or 09 UTC) time of fog and the meteorological conditions of high relative humidity $(>90 \%)$, relatively colder surface temperature and weak winds at the surface, all the cases can be classified as radiation fog. The focus in each case is on the midnight period (18 UTC) to afternoon (09 UTC), because this can provide an insight into the behaviour of two models under the conditions susceptible for fog formation. The model forecasts from $18 \mathrm{UTC}$ (18 h forecast) of the foggy day to 09 UTC of the next day (33 h forecast) were compared with observations. This also allows us to evaluate the model performance at longer lead times beyond $24 \mathrm{~h}$. The verification was carried out for visibility, relative humidity and temperature at $1.5 \mathrm{~m}$ and wind speed at $10 \mathrm{~m}$. The mean errors in the model forecast were also computed for all the variables.

\section{Results and discussion}

\section{Performance of the models in three categories of visibility}

Figure 2 shows the relationship between the observed and predicted values of visibility from the models under clear, poor and very poor visibility conditions. None of the models is able to predict visibility values below $200 \mathrm{~m}$. The values of visibility predicted by DM are always smaller than those predicted by NCUM-G under all conditions. In clear conditions, the observed visibility ranges from 1 to $6 \mathrm{~km}$, whereas the values of visibility predicted by the models do not extend beyond $3 \mathrm{~km}$. A good agreement is found between the observed and predicted values of visibility up to $3 \mathrm{~km}$ (Figure $2 a$ ). In the case of poor (Figure $2 b$ ) and very poor (Figure $2 c$ ) visibility conditions, both the models highly over-predict the values of observed visibility.

The total 674 observations during three months were used to compute the scores for $12-33 \mathrm{~h}$ forecast from NCUM-G and DM. Figure 3 gives the scores of these two models for three thresholds of visibility corresponding to 


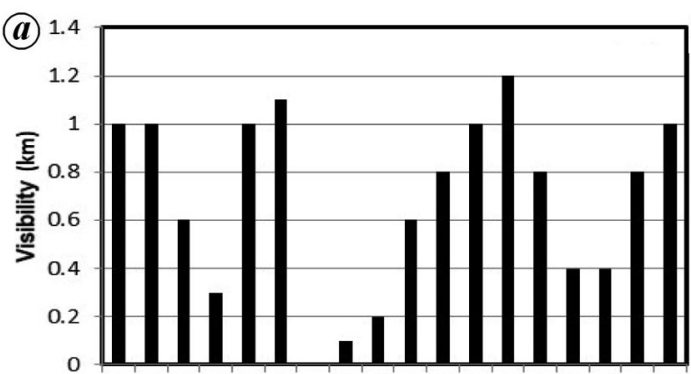

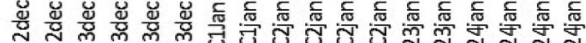

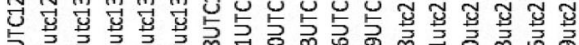

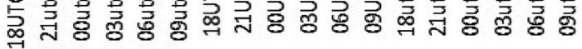

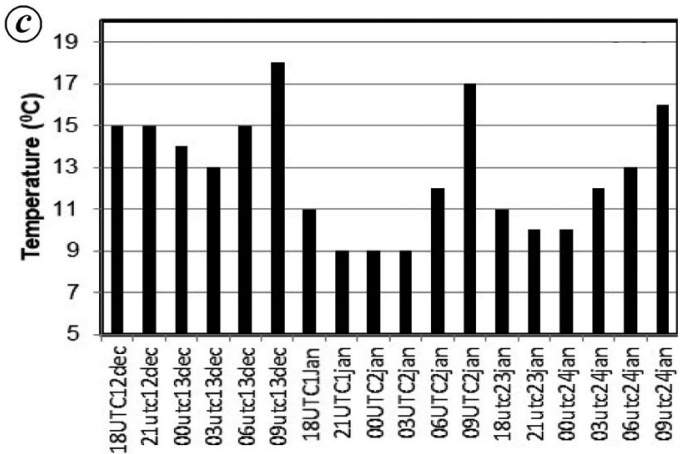

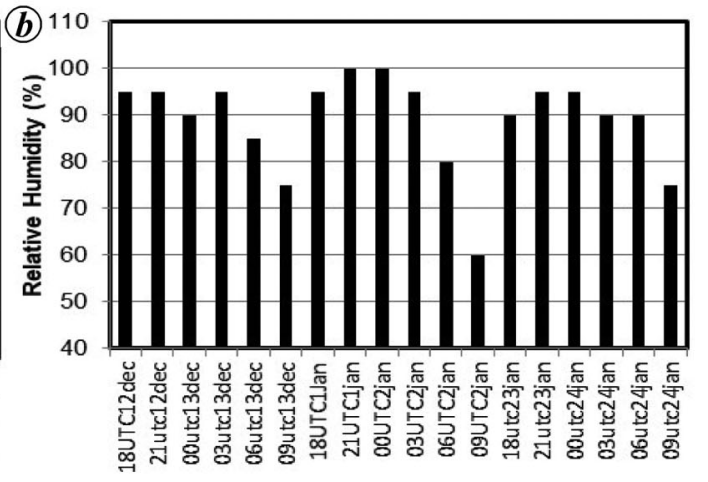

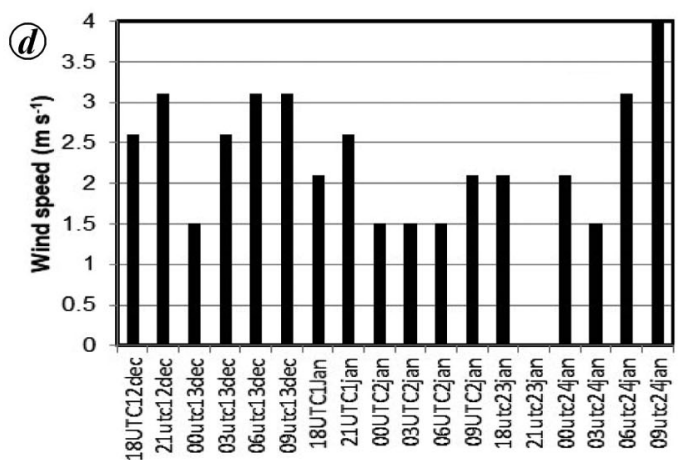

Figure 1. Observations of (a) visibility, $(\boldsymbol{b})$ relative humidity, $(\boldsymbol{c})$ temperature and $(\boldsymbol{d})$ wind speed for the selected cases of fog.

(a)

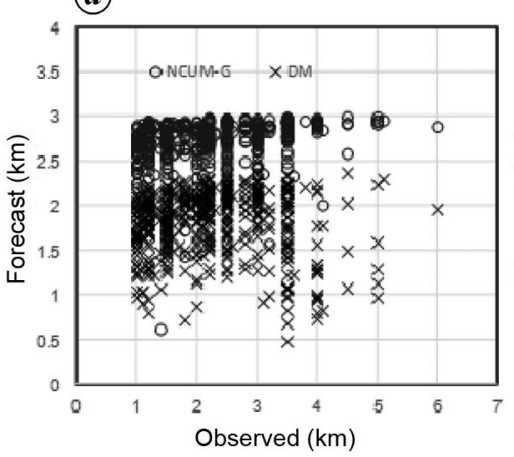

(b)

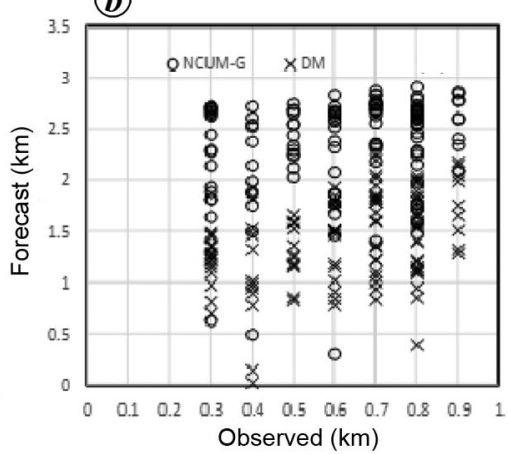

(c)

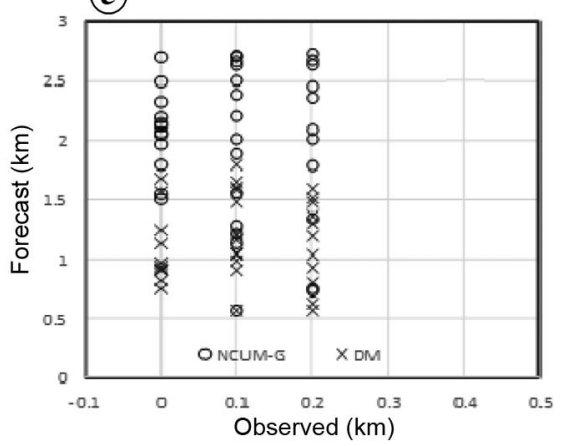

Figure 2. Scatter plot of observed and predicted values of visibility from NCUM-G and DM for (a) clear conditions, $(\boldsymbol{b})$ poor visibility conditions and $(c)$ very poor visibility conditions.

clear, poor and very poor conditions. The scores indicate that clear conditions are best predicted by both the models with highest value of POD and lowest value of FAR (Figure $3 a$ ). The value of FBI under clear conditions is more than 1 , which also indicates that these conditions are correctly predicted by both the models. Under poor visibility conditions, the values of POD and FBI are higher for DM than for NCUM-G. However, DM is not able to correctly predict visibility for all the hours and gives a significant value of FAR (Figure $3 b$ ). The value of POD is zero under very poor visibility conditions for both the models (Figure $3 c$ ). Also, the value of FAR is higher for DM (1.0) than NCUM-G (0.0) in these conditions.
The performance of DM is better than NCUM-G in predicting the poor visibility conditions, but it gives a smaller number of hits and more false alarms. It failed to predict the very poor visibility conditions. Thus, DM has a low potential of predicting poor visibility conditions, and has the tendency of producing more false alarm in both poor and very poor visibility conditions.

\section{Performance of the models during selected cases of} fog

As discussed earlier, all the observations suggest the formation of radiation fog in the three days. To evaluate the 

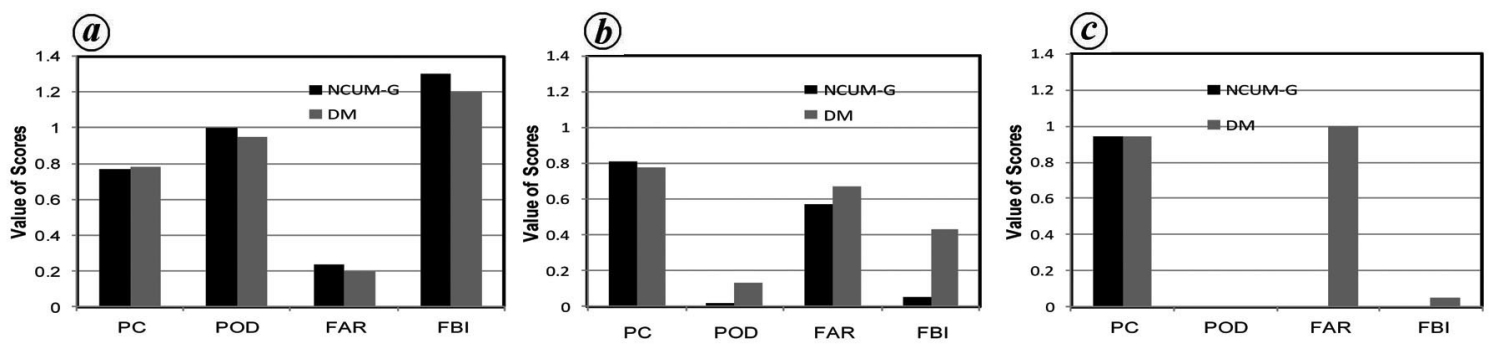

Figure 3. Scores of NCUM-G and DM in (a) clear conditions, $(\boldsymbol{b})$ poor visibility conditions and (c) very poor visibility conditions.

performance of these models in reproducing the observed values of visibility and the meteorological variables, a comparison between observed and predicted values of visibility, relative humidity and temperature at $1.5 \mathrm{~m}$ and wind speed at $10 \mathrm{~m}$ is shown in Figure 4 for the three cases. For the first case (12-13 December), the predicted values of visibility from DM are closer to observations than those predicted by NCUM-G (Figure $4 a$ ). None of the models is able to predict the observed values of visibility corresponding to very poor conditions in the second case (1-2 January). The predicted values of visibility from DM are always greater than $1 \mathrm{~km}$, whereas NCUM$\mathrm{G}$ predicts visibility close to the observations at 00 and 03 UTC of 2 January. For the third case, observed values of visibility are under-predicted by DM from 18 UTC of 23 January to 03 UTC of 24 January, and over-predicted by NCUM-G for all the hours, except one at 21 UTC of 23 January (Figure $4 a$ ). The observed visibility is found less than $1 \mathrm{~km}$ during daytime (06-09 UTC), which is not predicted by any of the models.

Figure $4 b$ compares the observed and predicted values of relative humidity for the same days. In the first case (12-13 December), the values predicted by NCUM-G are higher than those predicted by DM and those based on the observations. The values of relative humidity predicted by NCUM-G are found comparable to observations for majority of hours in the second case (1-2 January), whereas for the same case, DM predicts very low values (less than 90\%) of relative humidity. For the third case, the values of relative humidity predicted by both the models are comparable and higher than observations.

The temperature at $1.5 \mathrm{~m}$ and wind speed at $10 \mathrm{~m}$ are compared with observations in Figure $4 c$ and $d$ respectively. The observed temperature is under-predicted by both the models at night-time for the first case (12-13 December), whereas in the night of the second case (1-2 January), the temperature predicted by NCUM-G shows good agreement with observations and is over-predicted by DM. Further during night-time of the third case, observed temperatures are comparable to those predicted by NCUM-G and under-predicted by DM (Figure $4 c$ ).

Figure $4 d$ compares the observed and predicted wind speed at $10 \mathrm{~m}$. The values of wind speed are highly under-predicted by DM in comparison to the observed values in all the cases. However, NCUM-G predicts higher values of wind speed than DM for majority of hours (Figure $4 d$ ). Another important point is that DM predicts values of wind speed less than $2 \mathrm{~m} \mathrm{~s}^{-1}$ for majority of hours, whereas NCUM-G predicts wind speed values more than $3 \mathrm{~m} \mathrm{~s}^{-1}$.

Table 3 shows the mean error for the forecasted values of visibility, relative humidity and temperature at $1.5 \mathrm{~m}$ and wind speed at $10 \mathrm{~m}$ by NCUM-G and DM for three selected cases. Both the models overpredict the observed values of visibility, and the magnitude of overprediction is less in DM than NCUM-G. This may be attributed to the parameterization scheme of visibility used in the models. The prediction of visibility depends on the prediction of relative humidity and aerosol content. Depending on the values of relative humidity, the aerosol particles in the model get hydrated and become fog droplets, thereby reducing the visibility. The number of aerosols given in terms of mass mixing ratio is a fixed quantity and is higher in DM than in NCUM-G. Due to high value of mass mixing ratio in $\mathrm{DM}$, it always gives low visibility than NCUM-G. At very high values of relative humidity $(>98 \%)$, when the aerosols in the models become activated fog droplets, the number of fog droplets in DM becomes much higher than that in NCUM-G and under-predicts the observed values of visibility. Thus, DM sometimes performs better than NCUM-G in predicting visibility, but it also produces very low visibility conditions which are not actually observed. None of the models is able to predict the low visibility conditions below $1 \mathrm{~km}$ during daytime as the values of relative humidity predicted by both the models remain less than $90 \%$. Further, some interesting differences are seen between the two models in predicting the meteorological conditions conducive for formation of radiation fog.

The values of relative humidity and wind speed are better predicted by NCUM-G than by DM. The reason of higher under-prediction of wind speed values by DM in comparison to those by NCUM-G may be the blended PBL scheme used for turbulent parameterization in DM. The use of 3D-Smagronisky scheme increases the drag which reduces the wind speed in $\mathrm{DM}^{36}$. Both the models show colder bias at a forecast lead time of 24 and $27 \mathrm{~h}$. 

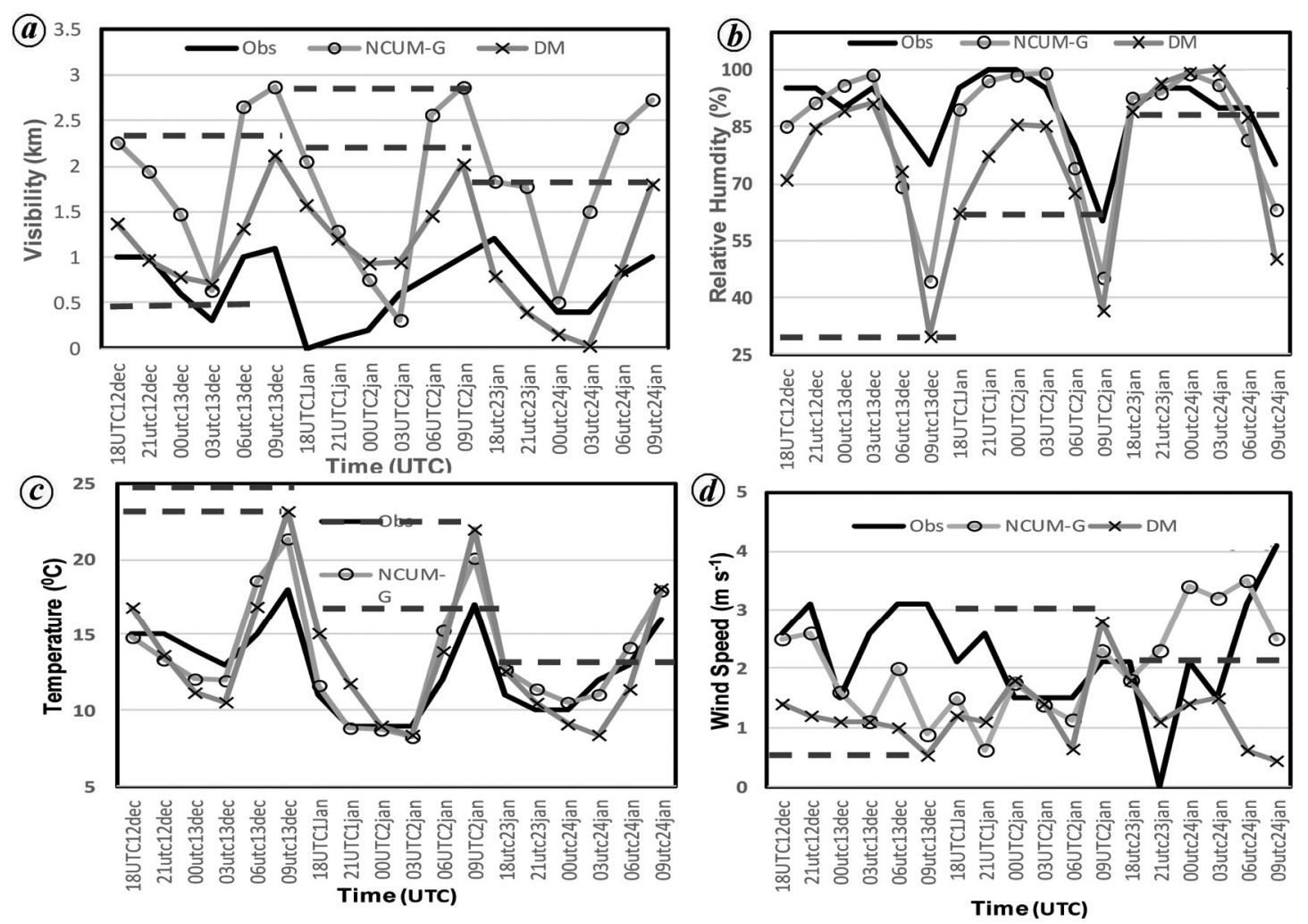

Figure 4. Comparison of observed and predicted (a) Visibility, (b) relative humidity, $(\boldsymbol{c})$ temperature and (d) wind speed using a NCUM-G and DM for three selected cases.

This is due to underestimation of surface temperature at these forecast lead times. This underestimation is higher in DM than in NCUM-G, and thus it predicts colder night-time minimum temperatures. This indicates that the performance of NCUM-G is better than DM in predicting the values of meteorological variables responsible for formation of radiative fog. It is also worth noting that the sample size is small and thus significance of small differences between the models should not be overstated. An extensive study with a greater number of cases of radiative fog with DM may further help in understanding the role of turbulent parameterization scheme in the evolution of fog.

\section{Summary and conclusion}

The present study focuses on the performance of coarse and high-resolution models to predict visibility under clear conditions with no fog as well as in poor and very poor visibility conditions corresponding to dense and very dense fog respectively. The statistical comparison presented here demonstrates that clear conditions are best predicted by both the models. The high-resolution DM shows better skill than NCUM-G in predicting poor visibility conditions, however, the statistical scores remain low. None of the models is able to predict the very poor visibility conditions. In addition, DM tends to under-predict the visibility values due to which it produces more false alarms than NCUM-G in all the conditions. Detailed analysis of three case studies presented here reveals that the main reason for these differences relates to the difference in the constant value of aerosol content used in the visibility parameterization scheme of the two models. Consistent with earlier results ${ }^{14}$, the prediction of visibility from DM is also largely influenced by correct prediction of relative humidity and the number of aerosols. The performances of the two models are comparable in predicting visibility during fog. The enhanced resolution and use of improved turbulent parameterization scheme in DM could not contribute much in predicting correct visibility conditions. The improvement in visibility parameterization scheme by including the prognostic aerosol can help in predicting more accurate values of visibility.

The meteorological conditions with high relative humidity and low wind speed, favourable for the formation of radiative fog are better predicted by NCUM-G than DM. This is a preliminary study on the performance of the high-resolution model in predicting visibility for dense and very dense fog. The grid length of DM is still not at a scale that can explicitly resolve the turbulent processes in the stable BL and the model heavily depends on the turbulence parameterization scheme. Further work 
with a greater number of observations in radiative fog should be carried out to analyse the effect of high resolution and the improved parameterization scheme of turbulence in DM in predicting meteorological conditions conducive for the formation of radiation fog.

1. Kulkarni, R. G., Wintertime fog in Delhi and its effect on aviation economy. M.Sc. Project Report Submitted to Savitribai Phule University, Pune, 2016.

2. Basu, S. C., Fog over upper Assam. Indian J. Meteorol. Geophys., $1957,8,67-71$

3. Natrajan, G. and Banerji, R. C., Fog over Agartala Airfield. Indian J. Meteorol. Geophys., 1959, 10, 161-167.

4. Gupta, R. K., On techniques of forecasting fog/stratus over the Dundigal airfield of Hyderabad. Mausam, 1987, 38, 401-406.

5. Tulsidas, A. and Mohapatra, M., Analysis and forecasting fog over Bangalore Airport. Mausam, 1998, 49(1), 135-142.

6. Dimri, A. P. et al., Western disturbances: a review. Rev. Geophys., 2015, 53, 225-246; https://doi.org/10.1002/2014rg000460.

7. Jenamani, R. K., Alarming rise in fog and pollution causing a fall in maximum temperature over Delhi. Curr. Sci., 2007, 93, 314 322

8. Pithani, P. et al., WRF model sensitivity to choice of PBL and microphysics parameterization for an advection fog event at Barkachha, rural site in the Indo-Gangetic basin. India Theor. Appl. Climatol., 2018; https://doi.org/10.1007/s00704-018-2530-5.

9. Payra, S. and Mohan, M., Multirule based diagnostic approach for the fog predictions using WRF modelling tool. Adv. Meteorol., 2014; https://doi.org/10.1155/2014/456065.

10. Goswami, P. and Sarkar, S., An analogue dynamical model for forecasting fog-induced visibility: validation over Delhi. Meteorol. Appl., 2017, 24, 360-375.

11. Petersen, C. and Nielsen, N. W., Diagnosis of visibility in DMIHIRLAM. Scientific Report 00-11. DMI, Copenhagen, Denmark, 2000

12. Stoelinga, M. T. and Warner, T. T., Nonhydrostatic, mesobetascale model simulations of cloud ceiling and visibility for an East Coast winter precipitation event. J. Appl. Meteorol., 1999, 38, 385-404.

13. Richard, M. Chmielecki, and Adrian, E. R., Probabilistic visibility forecasting using Bayesian model averaging. Mon. Weather Rev., 2011, 139, 1629-1636.

14. Haywood, J. et al., Prediction of visibility and aerosol within the operational Met Office Unified Model. II. Validation of model performance using observational data. Q. J. R. Meteorol. Soc., 2008, 134, 1817-1832.

15. Aditi, George, J. P., Gupta, M. D., Rajagopal, E. N. and Basu, S., Verification of visibility forecasts from NWP model with satellite and surface observations. Mausam, 2015, 66, 603-616.

16. Aditi, S., George, J. P. and Iyengar, G. R., Prediction of fog/ visibility over India using NWP model. J. Earth Syst. Sci., 2018, 127, 26; https://doi.org/10.1007/s12040-018-0927-2.

17. Jayakumar, A., Rajagopal, E. N., Boutle, I. A., George, J. P., Mohandas, S., Webster, S. and Aditi, S., An operational fog prediction system for Delhi using $330 \mathrm{~m}$ Unified Model. Atmos. Sci. Lett., 2018, 19, e796.

18. George, J. P., Indira Rani, S., Jayakumar, A., Mohandas, S., Mallick, S., Lodh, A. and Rajagopal, E. N., NCUM data assimilation system, 2016, NMRF/TR/01/2016.

19. Davies, T., Cullen, M. J. P., Malcolm, A. J., Mawson, M. H., Staniforth, A., White, A. A. and Wood, N., A new dynamical core for the Met Office's global and regional modelling of the atmosphere. Q. J. R. Meteorol. Soc., 2005, 131, 1759-1782.

20. Edwards, J. M. and Slingo, A., Studies with a flexible new radiation code, Part 1: choosing a configuration for a large-scale model. Q. J. R. Meteorol. Soc., 1996, 122, 689-719.

21. Wilson, D. R. and Ballard, S. P., A microphysically based precipitation scheme for the UK Meteorological Office Unified Model. Q. J. R. Meteorol. Soc., 1999, 125, 1607-1636.

22. Lock, A. P., The numerical representation of entrainment in parametrizations of boundary layer turbulent mixing. Mon. Weather Rev., 2001, 129, 1148-1163.

23. Brown, A. R., Beare, R. J., Edwards, J. M., Lock, A. P., Keogh, S. J., Milton, S. F. and Walters, D. N., Upgrades to the boundarylayer scheme in the Met Office numerical weather prediction model. Bound. Layer Meteorol., 2008, 128, 117-132.

24. Smith, R. N. B., A scheme for predicting layer clouds and their water content in a general circulation model. Q. J. R. Meteorol. Soc., 1990, 116, 435-460; doi:10.1002/qj.49711649210.

25. Best et al., The Joint UK Land Environment Simulator (JULES), model description - Part 1: Energy and water fluxes. Geosci. Model Dev., 2011, 4, 677-699.

26. Lock, A. P., Brown, A. R., Bush, M. R., Martin, G. M. and Smith, R. N. B., A new boundary layer mixing scheme. Part I: scheme description and single-column model tests. Mon. Weather Rev., 2000, 128, 3187-3199.

27. Boutle, I. A., Eyre, J. E. J. and Lock, A. P., Seamless stratocumulus simulation across the turbulent gray zone. Mon. Weather Rev., 2014, 142, 1655-1668.

28. Lilly, D. K., The representation of small-scale turbulence in numerical simulation experiments. In Proceedings of IBM Scientific Computing Symposium on Environmental Sciences (ed. Goldstine, H. H.), NY, USA, 1967, pp. 195-210.

29. Wyngaard, J. C., Toward numerical modeling in the "terra incognita'. J. Atmos. Sci., 2004, 61, 1816-1826.

30. Beare, R., A length scale defining partially-resolved boundarylayer turbulence simulations. Bound. Layer Meteorol., 2014, 151, 39-55; doi:10.1007/s10546-013-9881-3.

31. Efstathiou, G. A. and Beare, R. J., Quantifying and improving sub-grid diffusion in the boundary-layer grey zone. Q. J. R. Meteorol. Soc., 2015, 141, 3006-3017; doi:10.1002/qj.2585.

32. Clark, P. A., Harcourt, S. A., Macpherson, B., Mathison, C. T., Cusack, S. and Naylor, M., Prediction of visibility and aerosol within the operational Met Office Unified Model. I: Model formulation and variational assimilation. Q. J. R. Meteorol. Soc., 2008, 134, 1801-1816

33. Koschmeider, H., Therie der horizontalen sichtweite. Beitr. Phys. Freien Atmos., 1924, 12, 33-35.

34. Singh R. D., Singh, D. and Dube, R. K., Climatology and trend of visibility of IGI Airport, New Delhi and their use in forecasting. In Proceedings of the National Symposium TROPMET-1999, India Meteorological Society, Chennai, pp. 623-628.

35. Jenamani, R. K. and Tyagi, A., Monitoring fog at IGI airport and analysis of its runway-wise spatio-temporal variations using Meso-RVR network. Curr. Sci., 2011, 100, 491-501.

36. Boutle, I. A., Finnenkoetter, A., Lock, A. P. and Wells, H., The London model: forecasting fog at $333 \mathrm{~m}$ resolution. Quart. J. R. Meteorol. Soc., 2016, 142, 360-371; https://doi.org/10.1002/ qj.2656.

Received 20 May 2020; revised accepted 5 November 2020

doi: $10.18520 /$ cs/v120/i4/676-683 